Literatura y Lingüística $\mathrm{N}^{\circ} 24$

ISSN 0716-5811 / pp. 199-223

\title{
Divergencias y encuentros locutivos en el habla de dos grupos sociales*
}

\author{
César Marcelo Díaz Pacheco**
}

\section{Resumen}

Es un hecho evidente que las personas que forman parte de una comunidad idiomática determinada no hablan del mismo modo. En este sentido, diversos estudiosos han considerado muchas diferencias, por lo cual se han abocado a buscar su origen en diferentes factores: las características sociales del emisor (como ser hombre o mujer, joven o adulto, etc.), las características sociales del interlocutor (por ejemplo, tener más o menos estatus que el emisor), el entorno geográfico en el cual se desarrolla la interacción, etc. En este marco, la presente investigación pretende analizar las diferencias y similitudes de tipo fraseológico existentes en la forma de habla de dos grupos sociales distintivos de la Quinta Región de Chile. Para ello, se contrastaron dos grupos de estudiantes de enseñanza media, pertenecientes a un establecimiento municipal y un establecimiento subvencionado de la zona, respectivamente. Para estos fines, se propone un nivel de variación sociolingüística que, aparentemente, no ha sido muy estudiado: el nivel de la variación fraseológica.

Palabras clave: locuciones, frases hechas, variables sociales, variables lingüísticas

\section{Disagreements and locutionary similarities in the speech of two social groups}

\begin{abstract}
It is a well known fact that people who belong to a particular linguistic community do not speak at a similar way. In this sense, different studies have considered a lot of differences, for which reason, they look for its origin in differents factors : social characteristics of the speaker (like being man or woman, young or adult, and so forth), social characteristics of the interlocutors (for example, to have more or less status that the speaker), the geographic enviroment in which the interaction is developed, and so on. Whithin this framework, this investigation tries to analyze the differences and similarities of phraseological type that exist in the form of speaking of two distinctive social groups of the Fifth Region of Chile. For that reason, two groups of secondary school, one of them belonging to a municipal institution and the other one to a state-subsidiezed institution, were respectively compared. Consequently, a level of sociolinguistic variation is proposed that, apparently, it has not attracted too mucsh attention: the level of phraseological variation.
\end{abstract}

Key words : idioms, set phrases, social variables, linguistic variables

Recibido: 06-04-2011 Aceptado: 09-05-2011

\footnotetext{
Investigación realizada en el marco del proyecto FONDECYT 1070333

** Licenciado en Educación y Licenciado en Lengua y Literatura Hispánica. Docente. Pontificia Universidad Católica de Valparaíso. ILCL. Email: cesar.diaz@ucv.cl
} 
Divergencias y encuentros locutivos en el habla de dos grupos sociales / César Marcelo Díaz Pacheco

\section{Introducción}

Es un hecho evidente que los sujetos que forman parte de una comunidad idiomática determinada no hablan del mismo modo. Desde esta perspectiva, la sociolingüística se ha encargado de describir los factores que inciden en el origen de estas diferencias, intentando explicar las razones que justifican y dan cuenta de su funcionalidad en la construcción de identidades sociales. Asimismo, esta disciplina analiza cómo la organización de la variación lingüística de una comunidad se presenta condicionada por un tipo de jerarquización social (en clases, en grupos de edades y otras categorías de tipo socio-cultural).

Esta investigación pretende analizar las diferencias y similitudes de tipo fraseológico existentes en la forma de habla de dos grupos socioeconómicos de la ciudad de Valparaíso. Para ello, se propone un nivel de variación sociolingüística que -aparentemente- no ha sido muy estudiado: el nivel de la variación fraseológica.

En la primera parte, se presentará el concepto de variación y sus respectivos niveles. Posteriormente, se desarrollará la noción de fraseología y sus clasificaciones. Finalmente, se mostrarán las variables sociales que se utilizaron en este trabajo y los conceptos de variación social del uso del lenguaje y prestigio lingüístico.

En la segunda parte, se dará cuenta de la metodología utilizada, la cual intentará comparar la producción fraseológica de sujetos pertenecientes a dos estratos socio-económicos diferentes. Para ello, se contempla registrar la producción fraseológica de los dos estratos sociales distintivos, analizar la producción lingüística fraseológica de cada grupo y medir la comprensión fraseológica que tienen los sujetos de una clase social sobre la producción fraseológica de la otra, respectivamente. Junto con esto, se explicitará la descripción de los instrumentos utilizados y de los sujetos participantes en este estudio. Así, daremos paso al análisis de los resultados.

Por último, se presentarán las conclusiones arrojadas en este trabajo, en conjunto con las limitantes y proyecciones en base a lo investigado.

\section{Marco Teórico}

\section{I.1. Concepto de variación}

La lengua no es un sistema homogéneo, sino que es intrínsecamente variable. Podemos usar distintas expresiones para referirnos a los mis-

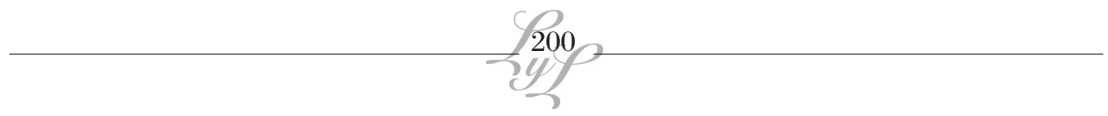


mos conceptos, así por ejemplo, decir perro o can. También, podemos utilizar distintos pronombres en distintas regiones y el español vosotros es reemplazado por ustedes en Latinoamérica. Coseriu (1973) plantea que la lengua varía porque no está hecha, sino que se está haciendo constantemente y se presenta como un sistema de posibilidades, entre las cuales cada comunidad de usuarios elige las que considera más adecuadas.

Desde esta perspectiva, podemos enfatizar que la lengua se desarrollará y manifestará de modo variable. Con esto decimos que los hablantes acuden consciente o inconscientemente a diversos elementos lingüísticos para expresar cosas distintas; y que, a la vez, existe la posibilidad de usar elementos lingüísticos diferentes para decir unas mismas cosas (Moreno, 1998).

De este modo, una teoría de la variación debería explicar el origen y el uso de las unidades que varían, cambian y alternan. Dejando a un lado la variación motivada en factores internos, las fuentes de variación que generalmente se han considerado en lingüística son la historia, la geografía, la sociología, la situación comunicativa y la psicología. Entonces, podemos hablar de variación histórica, geo-lingüística, socio-lingüística y estilística (Moreno, 1998). De esta forma, al elemento o unidad lingüística que puede presentarse de modos diversos se le da el nombre de variable lingüística. Así, una variable lingüística es un conjunto de expresiones de un mismo elemento y cada una de las expresiones de una variable recibe el nombre de variante lingüística (Moreno, 1998). Ejemplificando lo anterior, podemos decir que en Chile puede hablarse de un chiquillo o de un muchacho, en España se habla de chaval y en Argentina de pibe.

Adentrándonos en el proceso de identificación de un fenómeno de variación, los especialistas recurren al auxilio de disciplinas como la dialectología o la historia de la lengua, ya que es habitual que existan factores extra-lingüísticos implicados en la variación: la geografía (variación geo-lingüística), la historia (variación histórica), la extracción social del hablante o la situación comunicativa.

Con esto, podemos señalar que los factores que definen la aparición de unas variantes lingüísticas en ciertas circunstancias y de otras variantes en circunstancias diferentes responden a cuatro posibilidades (López, 1983:150):

a) Que las variantes vengan determinadas exclusivamente por factores lingüísticos.

b) Que las variantes vengan determinadas exclusivamente por factores sociales. 
Divergencias y encuentros locutivos en el habla de dos grupos sociales / César Marcelo Díaz Pacheco

c) Que las variantes vengan determinadas conjuntamente por factores lingüísticos y sociales.

d) Que las variantes no vengan determinadas por factores lingüísticos ni por factores sociales.

De las cuatro posibilidades señaladas, nos centraremos en a y c por ser las más idóneas para nuestro estudio. Cuando se comprueba que la variación lingüística está correlacionada con factores de naturaleza social, hablamos de variación sociolingüística. Pero antes de referirnos a ella, es necesario explicar cuáles son aquellos niveles de la lengua que pueden ser afectados por la variación.

\section{I.2. Niveles de variación}

Al hacer referencia a factores que determinan la aparición de variantes lingüísticas, decimos que estos fenómenos aparecen dentro de una comunidad de habla. Pero, iqué se entiende por Comunidad de habla?

Una Comunidad de habla está formada por un conjunto de hablantes que comparten (al menos) una lengua, un conjunto de normas y valores de naturaleza sociolingüística, unas mismas reglas de uso, un mismo criterio a la hora de valorar socialmente los hechos lingüísticos y unos mismos patrones sociolingüísticos. De este modo, los hispanohablantes de Chile y de España pertenecen a una misma comunidad idiomática, pero no a una misma comunidad de habla (López, 1983:150).

\section{a) Variación fonético-fonológica}

Este nivel establece que las variantes de un fonema no suponen, al alternar, ningún cambio de significado. Así, podemos ejemplificar la aparición en ciertas circunstancias de las variantes $[\mathrm{s}]$ o [ $\varnothing]$ del fonema /s/ implosivo del español no implica cambio semántico alguno (los aviones, lo avione); lo mismo ocurre con las variantes [r] y [l] del fonema

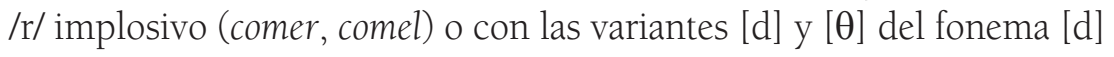
cuando aparece en posición final de la palabra (verdad, verdaz).

\section{b) Variación gramatical: morfología y sintaxis}

La variación sintáctica plantea el problema de las posibles diferencias de significado entre las variantes. A ello, puede añadirse que la variación sintáctica, muy frecuentemente, no está estratificada social ni estilísticamente, sino que viene determinada por factores netamente lingüísticos. 
Las variables de tipo morfológico son aquellas que afectan a elementos de la morfología, cuya variación rara vez implica los niveles del tipo pragmático y sintáctico. Serían variables de tipo morfológico las que se presentan a continuación:

No quisiste avisarme / No quisistes avisarme

Si quisiera lo podría hacer / Si quisiese lo podría hacer

Las variables de tipo categorial son aquellas que afectan, en muchos casos, a elementos de la morfología y, en casi todos, a la sintaxis, cuya variación implica a veces elementos de tipo semántico-pragmático. Ejemplos de este tipo podrían ser:

Me llaman para que yo redacte el informe / Me llaman para redactar el informe

Ella subió las escaleras muy rápida / Ella subió las escaleras muy rápido

Las variables de tipo funcional son aquellas que afectan a la sintaxis, de forma parcial a la morfología, y que no suelen estar correlacionadas con otros factores de naturaleza semántica. Por otro lado, como sucede con las variables de tipo morfológico, recurrentemente resultan determinadas por factores históricos, geográficos, sociolingüísticos y estilísticos, aunque no en todos los casos es así. Algunos ejemplos de estas variables son las siguientes:

Yo digo que esto es cierto / Yo digo de que esto es cierto

Ha habido muchos problemas / Han habido muchos problemas

\section{c) Variación Léxica}

El estudio de la variación léxica se enfrenta a los mismos problemas que la variación gramatical. En este sentido, es inevitable que nos surja la pregunta ¿qué se busca al estudiar la variación léxica? La respuesta no puede ser muy distinta de la que se ha dado en relación a los otros niveles: se intenta explicar el uso alternante de unas formas léxicas -normalmente sustantivos, verbos o adjetivos- en unas condiciones lingüísticas y extra-lingüísticas determinadas. Así, se pueden presentar unidades de distinto origen geo-lingüístico que han influido en una comunidad determinada (gorrión, pardillo), formas adscritas a niveles cultos o a niveles populares, así como a estilos más o menos formales (hijastro, entenado; encinta, preñada, embarazada; rasguño, raspón, rozón; burbuja, gorgorita, gargolita, farol) o formas tabúes o eufemísticas (axila, sobaco) entre otras posibilidades. De Igual modo, se busca también identificar 
Divergencias y encuentros locutivos en el habla de dos grupos sociales / César Marcelo Díaz Pacheco

el léxico característico de los diferentes grupos sociales: léxico juvenil, léxico profesional, léxico marginal, etc.

Los estudios de variación léxica han demostrado que en este tipo de variación participan principalmente factores extralingüísticos -rasgos sociológicos, situaciones, creencias y actitudes-, aunque también pueden estar implicados factores como el ritmo del habla, las repeticiones o la lengua de origen de las unidades léxicas. Así, no debemos dejar de tener en cuenta que, debido a su mayor dependencia del mundo social y cultural, el léxico se ve más afectado que las unidades gramaticales por fenómenos como la estereotipación y se halla sometido a restricciones contextuales más fuertes, por lo que aparece organizado de modo muy complejo desde el punto de vista pragmático (Almeida, 2003:75).

Desde esta perspectiva, las variables extralingüísticas actúan allí donde la lengua lo permite, no siendo casualidad que sea el nivel léxico -el más periférico o superficial- el más sujeto a los vaivenes históricos -el de mayor carga simbólica- donde estas variables parecen revelarse como las más determinantes.

\section{d) Variación Fraseológica}

Si bien, el nivel de variación relacionado con las formas fijas de la lengua -como son las colocaciones, las locuciones y los refranes- no ha recibido aparentemente atención de la sociolingüística, será uno de los objetivos de este trabajo considerarlo como un nivel de variación válido. Considerando los aportes de Corpas (1996) y Navarro (2004), en esta investigación se considerará el nivel fraseológico como un nivel que se encontraría entre lo léxico y lo gramatical propiamente tal.

\section{I.3. Concepto de Fraseología}

El término Fraseología se define como "el Conjunto de frases hechas, locuciones figuradas, metáforas y comparaciones fijadas, modismos y refranes, existentes en una lengua, en el uso individual o en el de algún grupo" (RAE, 1992).

Las frases hechas formarían parte de un conjunto de expresiones cuya interpretación semántica global es convencional y no se derivaría del análisis de sus componentes (Belinchón, 1999), es decir, su interpretación dependerá del conocimiento propio de una determinada cultura compartida por una comunidad lingüística. 
Por otra parte, las frases hechas (y los modismos, un tipo de frase hecha) también pueden ser consideradas metáforas semi-lexicalizadas, ya que conforman expresiones con un significado figurado prefijado por el uso que parece no guardar relación con su significado literal (Belinchón, 1999).

En lo que al concepto de metáfora se refiere, conviene señalar que en su definición básica se establece como un tropo que consiste en trasladar el sentido recto de las voces a otro figurado, en virtud de una comparación tácita (RAE, 1992). Se trata entonces de expresiones lingüísticas (sujeto, tópico o marco) cuya referencia traspasa elementos semánticos salientes hacia otra entidad de categoría diferente (foco, vehículo o predicado de la metáfora).

Los usos metafóricos pueden ser aplicados a afirmaciones del tipo: La vida es una lotería; símiles como mientras dormía, el mundo giraba a su alrededor, comparaciones explícitas como más perdido que el teniente Bello; a analogías entre sujeto y predicado de una oración más lento que el cáncer e incluso negaciones nadie es de piedra (Belinchón, 1999). En este sentido, podemos decir que las frases hechas son metáforas de uso habitual y frecuente en nuestro ámbito socio-cultural, de forma tipificada y bien delimitada (Chamizo, 2005).

Corpas (1997) y Navarro (2004) establecen una clasificación de estas unidades fraseológicas entre: locuciones, colocaciones y paremias.

Las locuciones se definen como una combinación estable de dos o más términos, que funcionan como elemento oracional y cuyo sentido unitario consabido no se justifica, sin más, como una suma del significado normal de los componentes (Casares, 1992, citado por Corpas, 1997). Asimismo, se caracterizan por su unidad de significación y fijación interna, equivalen a la lexía simple o al sintagma, pueden pertenecer a diversos tipos categoriales y cumplen diferentes funciones sintácticas (Navarro, 2004). En este sentido, implican una cohesión de tipo semántico y otra de tipo morfo-sintáctico. La primera se refleja en el carácter de unidad de significación en la lengua que presentan sus unidades, ya tengan significado compositivo (sano y salvo, loc. Sin lesión, enfermedad o peligro', DRAE) o traslaticio (al que le cayó el poncho, que se lo ponga. Loc. Decir algo haciendo referencia a alguna persona aludida). La segunda característica tiene relación en la aplicación de determinadas pruebas y operaciones formales, que comprueban no sólo la estabilidad formal de las locuciones, sino también su integridad semántica. Las principales pruebas aducidas son las siguientes (Corpas, 1996): 
Divergencias y encuentros locutivos en el habla de dos grupos sociales / César Marcelo Díaz Pacheco

1) Sustitución: Consiste en cambiar uno de los constituyentes de la unidad por un sinónimo, un hipónimo o hiperónimo, o por un tertium comparationis. El resultado es una secuencia gramatical y posible, pero que ya no conserva la cohesión semántica de la locución en cuestión: por ejemplo, dame una mano ("ayúdame") frente a regálame una mano.

2) Eliminación: Se suprime uno de los constituyentes, de forma que la secuencia resultante sea gramatical pero no conserve el significado de la unidad fraseológica. Por ejemplo, matar dos pájaros de un tiro ("Lograr dos cosas a través de un mismo hecho") frente a matar pájaros de un tiro. Tampoco se permiten adiciones: matar dos pájaros blancos de un tiro.

3) Deficiencias transformativas: Junto con las anteriores, se han aplicado otras pruebas subsidiarias, fundamentalmente a las locuciones verbales. Entre ellas figura el criterio de imposibilidad de reordenación de los elementos integrantes: dar gato por liebre ("engañar dando una cosa por otra de más valor") frente a dar liebre por gato. O el cambio de artículos: estirar la pata ("morir") frente a estirar una pata. Así, el orden de las palabras no puede someterse a los cambios permitidos por las combinaciones libres de palabras de estructura y categoría similares. De este modo, estirar la pata no permite transformación pasiva: El borracho estiró la pata <La pata fue estirada por el borracho>, frente a El borracho estiró el brazo <El brazo fue estirado por el borracho>.

En cuanto a su taxonomía, podemos decir que existen diferentes tipos de locuciones: las de estructura oracional (poseen verbo: caerse al litro, trabajar como chino) y las que carecen de ella (mosca/mosquita muerta), adjetivas (limpio de polvo y paja), adverbiales (con la boca abierta, a todas luces), conjuntivas (siempre y cuando, más le vale), prepositivas (con arreglo a, en torno a), clausales (hacérsele a alguien agua la boca).

Por otra parte, las colocaciones son combinaciones frecuentes de unidades léxicas fijas en la norma, las cuales se constituyen como unidades fraseológicas que, desde el punto de vista del sistema de la lengua, son sintagmas completamente libres, generadas a partir de reglas, pero que, al mismo tiempo, presentan cierto grado de restricción combinatoria determinada por el uso (cierta fijación interna). Este rasgo esencial distingue las colocaciones de las combinaciones libres de palabras (Liang, 1991-1992:154) Lo anterior, viene a decir que a fuerza de reproducir una combinación, los hablantes las reconocen 
como familiar, empleándolas como si se tratara de un fragmento prefabricado [...] Esto es, las colocaciones parecen almacenadas como unidades en el lexicón mental de los hablantes (Corpas, 1996). Ejemplo de colocaciones podemos mencionar: acariciar una idea, guerra fría, dinero sucio, etc.

Por último, las paremias o refranes forman parte por sí mismas de un mini texto debido a su autonomía material y de contenido (más vale pájaro en mano que cien volando), por lo que necesitan de un contexto verbal inmediato (Navarro, 2004). Se incluyen también la formulas rutinarias que tienen carácter de enunciado, pero se distinguen de las paremias por precisar de situaciones comunicativas específicas. Podemos mencionar ejemplos del tipo: expresiones de condolencia, saludos de despedida y festividades (ayudándole a sentir, felices pascuas).

De esta clasificación, nos interesan principalmente las locuciones y las colocaciones por ser las más idóneas para nuestra investigación. Asimismo, se partirá de la premisa en relación a las frases hechas como un nivel de la lengua, en el cual es posible estudiar la variación. Finalmente, cabe señalar que tanto las locuciones como las colocaciones consideradas serán propias de la forma de habla chilena. En otras palabras, se considerarán aquí expresiones que usan realmente los hablantes chilenos jóvenes, aunque esto no obsta que pudieren usarse en otros lugares de habla hispana.

\section{I.4. Variabilidad Socio-Lingüística}

La lengua es una forma de conducta social desarrollada y moldeada por los seres humanos que presenta diferenciaciones internas que corresponden a los parámetros que caracterizan a los diversos sub-grupos que constituyen el sistema socio-cultural. Las correlaciones variacionales entre lengua y sociedad han sido reconocidas desde siempre, pero los estudios cuantitativos del contexto social de la variación lingüística realizados en este último tiempo han demostrado que los factores sociales actúan de manera probabilística en la variación (Silva, 1989). De este modo, el contexto físico en que ocurre la comunicación, las relaciones entre los participantes y, tanto sus características adscritas (grupo generacional, sexo, etnicidad, casta, etc.) como adquiridas (nivel educacional, nivel socio-económico, etc.), han mostrado reflejarse de forma sistemática en comportamientos lingüísticos diferenciados. Esta covariación entre fenómenos lingüísticos y sociales es lo que define la variable sociolingüística (Silva, 2001). 
Divergencias y encuentros locutivos en el habla de dos grupos sociales / César Marcelo Díaz Pacheco

\section{I.5. Noción de clase social}

Si bien, no existe en la actualidad un consenso claro sobre el concepto de clase social, la sociología moderna ha optado por un enfoque multidimensional como una forma válida de descubrir diferencias relativas entre individuos, ya que es evidente que ciertos usos lingüísticos son más característicos de unos grupos (clases, niveles) que de otros; y que las diferencias sociolingüísticas aumentan conforme crece la distancia social entre los miembros de una comunidad (Moreno, 1998).

En este sentido, al ser nuestra investigación un estudio sociolingüístico inserto en poblaciones urbanas, uno de los patrones de covariación que emerge en forma distintiva es el que estratifica la población en diversos grupos que se han definido generalmente según el concepto de clase social. La pertenencia a un grupo social u otro influye tanto sobre la manera de hablar como sobre actitudes hacia estas diferentes maneras de hablar. El término estratificación social se emplea para referirse al orden jerarquizado de grupos de individuos dentro de una sociedad (Silva, 2001).

Así, es palmario que la distribución social de los usos lingüísticos funciona como factor decisivo en el desarrollo y la expansión de los cambios lingüísticos, y coordina frecuentemente con otras variables sociales, como la edad o el sexo. De este modo, al conjunto de características lingüísticas propias de un grupo, estrato o clase se le da el nombre de sociolecto (Labov, 1990). Desde este punto de vista, las diferencias jerárquicas vienen a reflejar desigualdades entre los grupos basadas en uno o más de los siguientes factores: nivel educacional, ocupación, la procedencia, los barrios y la edad.

\section{I.6. Variables Sociales}

En general, los estudios de sociolingüística que han incluido la variable clase social la han definido según criterios objetivos, razón por la cual el término factor socioeconómico se emplea con frecuencia en vez de clase social. De este modo, los niveles o variables sociales en los cuales se apoyará esta investigación serán los siguientes:

\section{a) Variable nivel educacional}

Educación, nivel de instrucción o de estudios son algunas de las denominaciones que ha recibido la variable que se refiere al tipo de formación académica conseguida por los hablantes. En este sentido, distintas disciplinas que han manifestado su preocupación por la lengua hablada, 
han comprobado que el nivel educativo de los hablantes define de forma directa y clara la variación lingüística. Es normal que las personas más instruidas hagan mayor uso de las variantes que son consideradas como más prestigiosas o que más se ajustan a la norma. Este hecho puede tener consecuencias importantes en el ámbito del cambio lingüístico (Moreno, 1998).

\section{b) Variable social ocupación}

Entre los modelos sociológicos -y sociolingüísticos- basados en la estratificación social, la profesión u ocupación es uno de los factores capaces de indicar la pertenencia de los individuos a unos grupos sociales o a otros; a la vez, el parámetro más ligado al concepto de estatus. La función social de una persona, la actividad que realiza en una comunidad está en relación directa con el lugar que ocupa en la jerarquía social y la valoración que de ella hacen los demás miembros de la comunidad.

\section{c) La procedencia y los barrios}

La procedencia geográfica del hablante y el barrio de residencia son variables pertinentes en lo que concierne a nuestra investigación, ya que aparte de ser un factor ligado al nivel socioeconómico de los individuos, el barrio es un elemento muy relacionado con la variable "procedencia geográfica", porque es habitual la concentración de individuos de un mismo origen en unas mismas zonas de las ciudades: existen barrios de tipo tradicional, en los que conviven personas cuya ascendencia es propia del lugar, y barrios receptores de inmigrantes.

\section{d) La edad}

La variable edad se muestra especialmente productiva en información en aquellos casos en que nos hallamos con algún tipo de estratificación relevante que nos indique que estamos ante un proceso de cambio. En este sentido, nos es importante este nivel ya que permite plantearnos y verificar la consistencia de afirmaciones de alcance más general que vinculan a ciertos grupos de edad con determinadas formas lingüísticas más o menos prestigiosas o más o menos vernáculas, entre otros factores.

\section{I.7. Variación Social del Uso del Lenguaje y Prestigio}

La existencia de actitudes lingüísticas supone la aceptación de que los seres humanos tienen la capacidad de evaluar su propia forma de hablar 
Divergencias y encuentros locutivos en el habla de dos grupos sociales / César Marcelo Díaz Pacheco

y el discurso de los demás a partir de la presencia de una serie de índices lingüísticos. Estos índices, que aparecen codificados socialmente bajo distintos atributos simbólicos, permiten a los miembros de cualquier comunidad extraer ciertas conclusiones sobre la condición social de los demás, sobre aspectos de la personalidad y sobre la misma situación comunicativa, todo lo cual nos lleva, en consecuencia, a planificar de un modo determinado la interacción (Almeida, 2003:187).

Desde esta perspectiva, podemos plantear que las actitudes hacia las diversas formas de habla que se observan en una comunidad se hallan en gran medida relacionadas con el estatus social de los grupos con los que se identifican tales variantes, de modo que aquellos rasgos lingüísticos que vendrían a caracterizar el habla de los grupos de más alto estatus serán considerados como más valiosos socialmente, más prestigiosos y correctos. A lo anterior, se denominará como Prestigio abierto (Almeida, 2003). Algunos ejemplos en nuestro país, podrían ser:

- La pronunciación tri: Asibilación del grupo /tr/; Articulación plena asibilada africada

- Partiré de acá atrás $(/ \mathrm{a} / / \mathrm{t} / / \mathrm{r} / / \mathrm{a} / / \mathrm{s} /)$

- Uso de un solo pronombre con perífrasis

- Le quiero dar una sorpresa

- Yo me tenía que ir a vacunar

- Uso de la ch: /ch/ africada sorda oral

- Una corrida de chanchos $(/ \mathrm{c} / \mathrm{h} / / \mathrm{a} / / \mathrm{n} / / \mathrm{c} / \mathrm{h} / / \mathrm{o} / \mathrm{s} /)$

Al contrario, los rasgos con los que aparecen identificados los grupos sociales con menor poder o estatus aparecen, de forma paralela, valorados como menos prestigiosos y a menudo, resultan estigmatizados. A lo anterior, se denominará como Prestigio encubierto. Algunos ejemplos podrían ser:

- La pronunciación fricativa arrastrada del grupo /tr/:

- Partiré de acá atrás (/a//t//r//r//a//s/)

- Reiteración de pronombres en las perífrasis verbales (duplicación pleonástica):

- Le quiero ir a darle una sorpresa.

- Yo me tenía que irme a vacunarme

- Uso fricativo del grupo /ch/:

- Una corrida de chanchos $(/ \mathrm{s} / / \mathrm{h} / / \mathrm{a} / / \mathrm{n} / / \mathrm{s} / / \mathrm{h} / / \mathrm{o} / / \mathrm{s} /)$ 
En relación con lo expuesto anteriormente, podemos sostener que en todo idioma existen ciertas palabras y oraciones cuyo empleo se halla restringido porque la sociedad considera prohibido «nombrar» la cosa directamente. Y como una palabra es un complejo de asociaciones y valoraciones afectivas, el hablante cambia aquellas palabras que evocan no sólo la «cosa» sino el conjunto de circunstancias que rodean y matizan el concepto. Así, el cáncer es sustituido por el mal, porque es una enfermedad mortal que no se menciona en casa del afectado, puesto que está cargada de juicios peyorativos. En este sentido, la palabra «prohibida» se denominará tabú lingüístico y eufemismo el término que la sustituye (Matus, 1999). Al respecto, Allan y Burridge (1991) sostendrán que el eufemismo se utiliza como una alternativa para expresiones que no se desean utilizar con el fin de evitar posibles disgustos, por la forma de habla que pueda ofender a la audiencia. Asimismo, el concepto de disfemismo será entendido como una expresión con connotaciones que son ofensivas tanto por aquello que denota como por la audiencia a la que va dirigida, por ejemplo, en Chile: "sobaco" es una forma disfémica de la palabra axila y hablar de los defectos femeninos a una audiencia de mujeres podría no ser muy apropiado. Debido a esto, los disfemismos en ciertos contextos pueden ser reemplazados por un término más neutro o una expresión eufemística (Allan y Burridge, 1991).

Este tipo de comportamiento, que ha sido interpretado como un claro reflejo de las relaciones de poder que existen entre los grupos sociales que componen una comunidad, no hace sino potenciar o acentuar las barreras sociales entre ellos (St. Clair, 1982). Muchas veces lo disfémico se asocia con los estratos socioeconómicos más bajos y lo neutro o eufémico con la forma de habla de los más altos. Sin embargo, ambos términos tienen valores para estilos distintos. Los disfemismos suelen mostrar mayor nivel de confianza e ir asociados a los usos informales del lenguaje, mientras lo eufémico se asocia a unas circunstancias menos íntimas y a un habla formal.

\section{Metodología}

\section{II.1. Objetivos e Instrumentos de Medición}

Este estudio se enmarca dentro de un enfoque descriptivo, pues su objetivo general es comparar las diferencias y similitudes en la producción fraseológica de sujetos pertenecientes a dos estratos sociales diferentes. Para ello, dentro de los objetivos específicos de esta investigación se 
Divergencias y encuentros locutivos en el habla de dos grupos sociales / César Marcelo Díaz Pacheco

contempla registrar la producción fraseológica de los dos estratos sociales distintivos, analizar la producción lingüística fraseológica de cada grupo y medir la comprensión fraseológica que tienen los sujetos de una clase social sobre la producción fraseológica de la otra, respectivamente. Para llevar a cabo dichos objetivos, se elaboraron dos instrumentos de medición. Uno de ellos fue de elicitación de locuciones y colocaciones y el otro de reconocimiento y evaluación actitudinal.

El test N1 está constituido por 10 ítems. En cada uno de ellos se le presenta al informante una situación que puede justificar el uso de determinadas frases hechas, en la cual se le pide al sujeto que diga todas las frases $u$ oraciones que recuerde y que puedan funcionar adecuadamente en esa situación específica. El test N2 está constituido por 10 ítems, en cada uno de ellos se le presenta al informante una situación. A continuación, se le presentan las frases que han sido registradas en el test anterior y que corresponden a informantes tanto de estratos medios como también bajos. Finalmente, se le pide al sujeto que señale si la conoce, en qué circunstancia la utilizaría y cómo la evalúa.

\section{II.2. Sujetos participantes en la muestra}

El primer instrumento fue aplicado a una muestra formada por cuatro jóvenes de entre 15 y 17 años de edad de estrato socio-económico bajo (dos mujeres y dos varones) residentes del sector Los Aromos en el cerro San Roque -grupo A-; en contraste con cuatro jóvenes de estrato socio-económico medio del Liceo Juana Ross de Edwards (dos mujeres y dos varones de la misma edad) -grupo B-. Ambos grupos insertos en la ciudad de Valparaíso. El segundo instrumento fue aplicado a 25 jóvenes de entre 15 y 17 años de edad de estrato socioeconómico bajo (15 mujeres y 10 hombres) estudiantes del Liceo Valparaíso B-29 -grupo A-; en contraste con 25 jóvenes de estrato socioeconómico medio del Liceo Juana Ross de Edwards (15 mujeres y 10 hombres de la misma edad) -grupo B-. Ambos grupos insertos en la ciudad de Valparaíso.

\section{3. Criterio de selección de las muestras}

La selección de los participantes responde a la finalidad de tomar muestras prototípicas de dos estratos socioeconómicos distintos. De los manuales de sociolingüística consultados (Moreno, 1998; Almeida, 2003) se puede inferir que la clase social no es un atributo evidente como lo sería el sexo de una persona. La dificultad se establece al determinar en forma clara qué se entenderá por estrato social y con qué

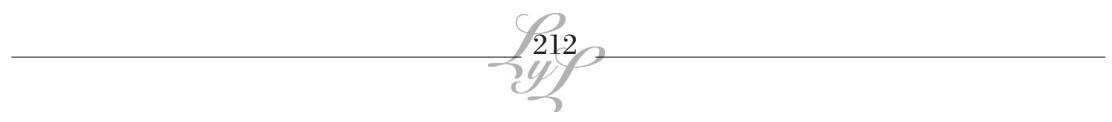


índices se medirá. A partir de allí, se establecerá que el estrato social de los sujetos se identificaría a partir de ciertos índices, con el fin de situar y categorizar socialmente a cada grupo participante. De este modo, en esta investigación se usaron como índices para ubicar a los informantes del test N1: la escolaridad de los padres, el barrio de residencia y el trabajo de los padres.

La selección de los sujetos pertenecientes a estos dos niveles obedeció a condiciones de disponibilidad de la muestra, por lo que fue más conveniente establecer en Valparaíso estos dos grupos socioeconómicos (en el caso del nivel socioeconómico alto, se presentó más difuso y difícil de identificar). En relación al estrato social de los informantes del test N2, se consideró el tipo de establecimiento educacional al cual pertenecen, debido a que la estructura del instrumento requería considerar grupos homogéneos como los que conforman los grupos-curso.

\section{Análisis de resultados}

\section{III.1. Conocimiento fraseológico de los sujetos participantes}

A continuación, se presenta el porcentaje de conocimiento que mostraron los sujetos sobre las frases hechas producidas por informantes de su propio estrato, por informantes del otro grupo social y aquellas que produjeron los informantes de ambos estratos a las cuales llamamos frases neutras. Dichas locuciones correspondieron tanto a frases prototípicas mantenidas en el tiempo como también a nuevos modismos usados actualmente en el habla juvenil. Algunos ejemplos seleccionados de la muestra: Estái peinando la muñeca (te volviste loco), Se te soltó un tornillo, Estiró la pata (falleció), Se echó a volar (se retiró), Andái Ponceando (andas de seductor).

La tabulación porcentual de las respuestas del Grupo A (compuesto por sujetos de clase baja) y del grupo B (compuesto por sujetos de clase media) respectivamente; arroja que no presentan mayores dificultades de conocimiento frente a la producción fraseológica tanto de los integrantes de su propio estrato socioeconómico, como del grupo al cual no pertenecen. 
Divergencias y encuentros locutivos en el habla de dos grupos sociales / César Marcelo Díaz Pacheco

Tabla 1. Porcentaje de conocimiento de frases hechas

\begin{tabular}{|c|l|c|}
\hline Sexo & Evaluación & Conocimiento Fraseológico \\
\hline h & clase baja sobre sí misma & $92,57 \%$ \\
\hline h & clase baja sobre clase media & $87,02 \%$ \\
\hline h & clase baja sobre frases neutras & $98,18 \%$ \\
\hline m & clase baja sobre sí misma & $93,30 \%$ \\
\hline m & clase baja sobre clase media & $82,18 \%$ \\
\hline m & clase baja sobre frases neutras & $96,96 \%$ \\
\hline h & clase media sobre clase baja & $80,27 \%$ \\
\hline h & clase media sobre sí misma & $76,21 \%$ \\
\hline h & clase media sobre frases neutras & $90,00 \%$ \\
\hline m & clase media sobre clase baja & $84,40 \%$ \\
\hline m & clase media sobre sí misma & $83,60 \%$ \\
\hline m & clase media sobre frases neutras & $93,30 \%$ \\
\hline
\end{tabular}

Como se puede observar en la tabla 1 , tanto los sujetos de estrato socio-económico medio como los de estrato socio-económico bajo reconocieron las frases hechas de sí mismos, neutras y del otro grupo por encima del 80\%. Una excepción la constituyen el grupo de los hombres de estrato medio quienes reconocen sólo el 76,21\% en uno de los casos. Tal vez, esto sea explicable porque los sujetos de estrato medio parecen ser más hábiles para manejar adecuadamente frases hechas más idiomatizadas y, por lo tanto, esa diversidad no sea reconocida por sus pares.

Si bien, en el gráfico 1 se pueden visualizar algunas diferencias porcentuales entre hombres y mujeres de los dos grupos (que podría expresar que los sujetos de estrato bajo tienen mayor conocimiento tanto de su forma de habla como de la perteneciente a los integrantes de estrato medio), no es mayormente significativa la distancia en la evaluación de uno u otro grupo social. Si observamos el gráfico, podemos ver que ningún grupo baja del 75\% de conocimiento fraseológico tanto, sobre su misma clase como también sobre la otra a la cual evalúan.

Gráfico 1. Porcentaje de conocimiento fraseológico
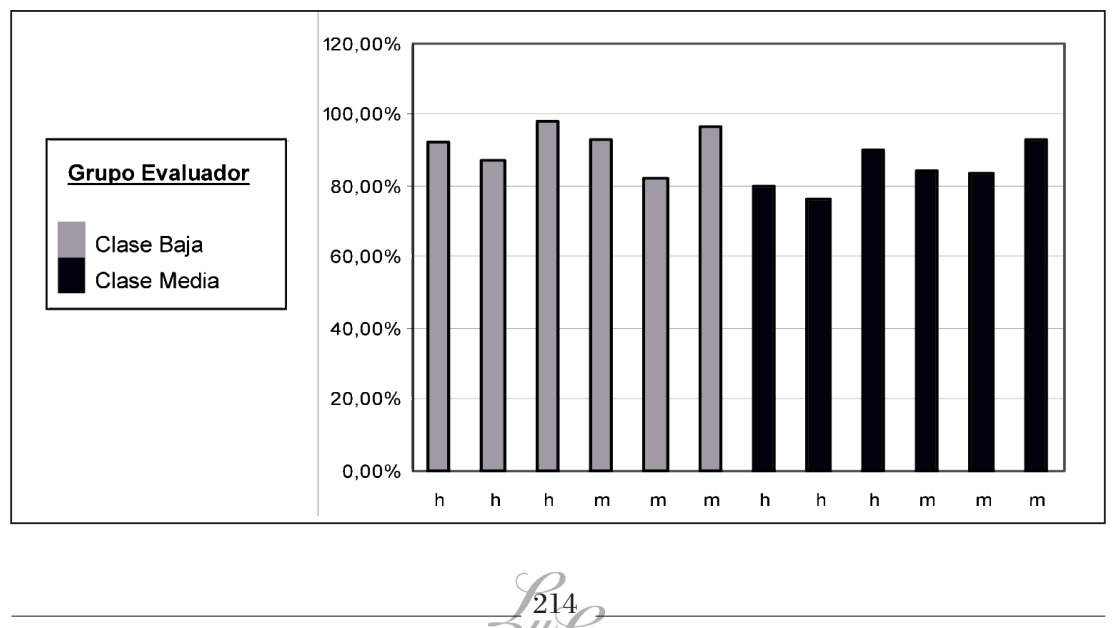


\section{III.2. Contextos de uso}

A continuación, presentamos los resultados de cómo juzgan estos jóvenes las frases hechas -en uso- que ellos producen y las que producen los integrantes del otro estrato social. Para ello, se les presentaron dos interlocutores diferentes: los amigos y el profesor. Los primeros remitirían a un contexto más informal, mientras que el segundo a un contexto más formal. Algunos ejemplos seleccionados de la muestra: Tengo el medio diente (tengo hambre), Te tienen de punto (te molestan demasiado), Es un caradura (es un descarado).

En un primer caso, podemos observar de acuerdo a los datos de la tabla 2 que en una situación de informalidad, ambos grupos sociales utilizarían en gran medida la producción fraseológica tanto de los integrantes de su propia clase social, como la de los sujetos pertenecientes a la otra.

Tabla 2. Porcentaje de uso en contexto informal

\begin{tabular}{|c|l|c|}
\hline Sexo & Evaluación & Uso con Amigos \\
\hline $\mathrm{h}$ & clase baja sobre sí misma & $97,22 \%$ \\
\hline $\mathrm{h}$ & clase baja sobre clase media & $93,47 \%$ \\
\hline $\mathrm{h}$ & clase baja sobre frases neutras & $98,14 \%$ \\
\hline $\mathrm{m}$ & clase baja sobre sí misma & $88,29 \%$ \\
\hline $\mathrm{m}$ & clase baja sobre clase media & $87,16 \%$ \\
\hline $\mathrm{m}$ & clase baja sobre frases neutras & $87,50 \%$ \\
\hline $\mathrm{h}$ & clase media sobre clase baja & $76,65 \%$ \\
\hline $\mathrm{h}$ & clase media sobre sí misma & $79,78 \%$ \\
\hline $\mathrm{h}$ & clase media sobre frases neutras & $79,79 \%$ \\
\hline $\mathrm{m}$ & clase media sobre clase baja & $76,31 \%$ \\
\hline $\mathrm{m}$ & clase media sobre sí misma & $79,94 \%$ \\
\hline $\mathrm{m}$ & clase media sobre frases neutras & $70,12 \%$ \\
\hline
\end{tabular}

Como se observa en la tabla anterior y en el gráfico 2, podemos notar que ninguno de los dos grupos baja del 70\% de uso, lo cual indicaría que tanto el grupo de estrato bajo como el de estrato medio utilizaría con frecuencia las frases hechas pertenecientes a los sujetos de su grupo como al del otro cuando tiene como interlocutor a un amigo, y por ello, en un contexto más informal. 
Divergencias y encuentros locutivos en el habla de dos grupos sociales / César Marcelo Díaz Pacheco

Gráfico 2. Uso con amigos

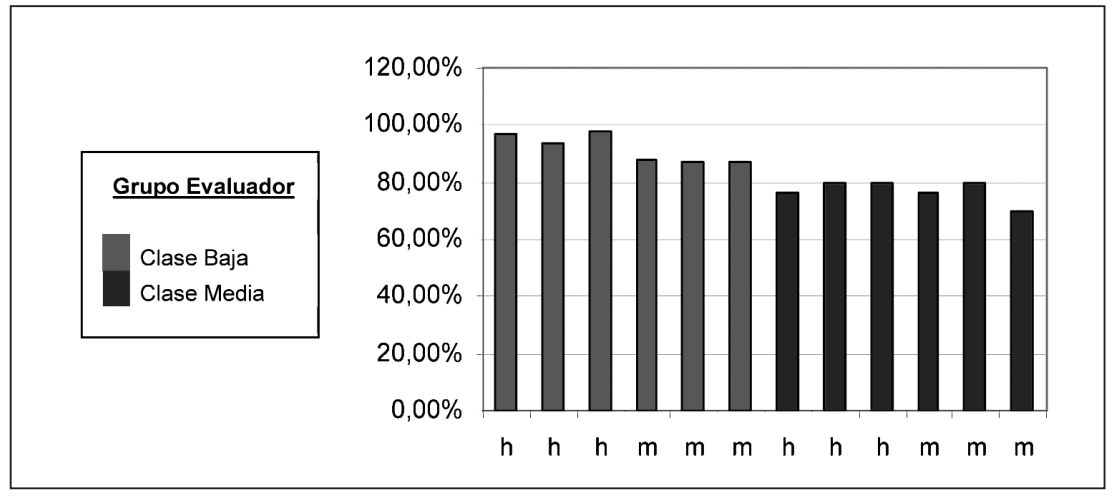

Sin embargo, en un segundo caso (situación formal de habla, considerando como destinatario al profesor) podemos observar en la Tabla 3 que se presentan diferencias considerables a la hora de hacer uso de la producción fraseológica de ambos grupos en esta situación.

Tabla 3. Porcentaje de uso en contexto formal

\begin{tabular}{|c|l|c|}
\hline Sexo & Grupos & Uso con profesor \\
\hline $\mathrm{h}$ & clase baja sobre sí misma & $67,90 \%$ \\
\hline $\mathrm{h}$ & clase baja sobre clase media & $62,42 \%$ \\
\hline $\mathrm{h}$ & clase baja sobre frases neutras & $56,48 \%$ \\
\hline $\mathrm{h}$ & clase media sobre clase baja & $26,55 \%$ \\
\hline $\mathrm{h}$ & clase media sobre sí misma & $39,00 \%$ \\
\hline $\mathrm{h}$ & clase media sobre frases neutras & $18,18 \%$ \\
\hline $\mathrm{m}$ & clase baja sobre sí misma & $52,97 \%$ \\
\hline $\mathrm{m}$ & clase baja sobre clase media & $56,63 \%$ \\
\hline $\mathrm{m}$ & clase baja sobre frases neutras & $45,63 \%$ \\
\hline $\mathrm{m}$ & clase media sobre clase baja & $15,57 \%$ \\
\hline $\mathrm{m}$ & clase media sobre sí misma & $20,74 \%$ \\
\hline $\mathrm{m}$ & clase media sobre frases neutras & $15,58 \%$ \\
\hline
\end{tabular}

De este modo, mientras los sujetos de estrato bajo en mayor porcentaje dicen que utilizarían estas frases hechas con el profesor, los jóvenes de estrato medio expresan que las usarían en menor medida en aquel contexto situacional. De esta manera, estos últimos parecerían considerar las frases hechas como más informales o menos adecuadas para utilizarlas con alguien que considerarían una autoridad. 
Grafico 3.Uso con el profesor

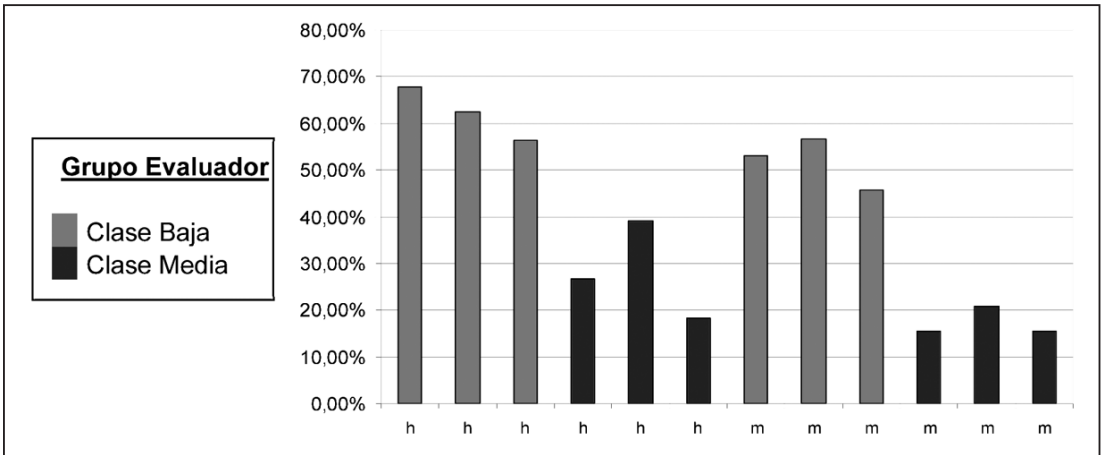

Como podemos ver, tanto las mujeres como los hombres de estrato bajo señalan en alrededor de un 50\% que usarían las frases hechas con su profesor. En el caso de los sujetos de clase media, ni los hombres ni las mujeres alcanzan esa cifra de uso fraseológico en esa situación particular que remite a lo formal.

\section{3. Eufemismos y disfemismos}

En este apartado, se consignan las respuestas evaluativas de los sujetos respecto de cuán groseras encontraban las frases hechas propias y las de sus pares del otro estrato. Aquí entra en juego la noción de disfemismo lingüístico unido a la noción de prestigio encubierto. Si bien, cuantitativamente los sujetos participantes no realizan evaluaciones disfémicas considerables tanto de su grupo como del otro al que no pertenecen, en términos porcentuales se observaría una cierta distancia entre ambas clases.

Tabla 4. Evaluación de disfemismo

\begin{tabular}{|c|l|c|}
\hline Sexo & Grupo A & Evalúa como grosera \\
\hline $\mathrm{h}$ & clase baja sobre sí misma & $32,09 \%$ \\
\hline $\mathrm{h}$ & clase baja sobre clase media & $4,96 \%$ \\
\hline $\mathrm{h}$ & clase baja sobre frases neutras & $27,70 \%$ \\
\hline $\mathrm{m}$ & clase baja sobre sí misma & $17,07 \%$ \\
\hline $\mathrm{m}$ & clase baja sobre clase media & $8,85 \%$ \\
\hline $\mathrm{m}$ & clase baja sobre frases neutras & $30,62 \%$ \\
\hline Sexo & Grupo B & Evalúa como grosera \\
\hline $\mathrm{h}$ & clase media sobre clase baja & $24,48 \%$ \\
\hline $\mathrm{h}$ & clase media sobre sí misma & $8,86 \%$ \\
\hline $\mathrm{h}$ & clase media sobre frases neutras & $35,35 \%$ \\
\hline $\mathrm{m}$ & clase media sobre clase baja & $41,44 \%$ \\
\hline $\mathrm{m}$ & clase media sobre sí misma & $9,05 \%$ \\
\hline $\mathrm{m}$ & clase media sobre frases neutras & $40,90 \%$ \\
\hline
\end{tabular}


Divergencias y encuentros locutivos en el habla de dos grupos sociales / César Marcelo Díaz Pacheco

Gráfico 4. Evaluación de disfemismo

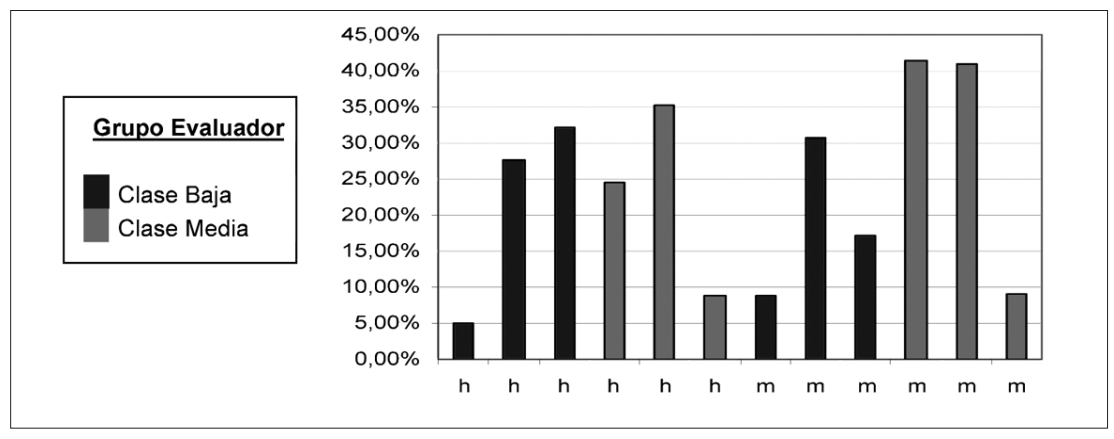

\section{III.4. Consideraciones finales}

Analizando los resultados de la muestra, podemos observar que los participantes del grupo B (estrato socio-económico medio) tienen mayor conciencia de las relaciones de simetría y del uso de la lengua en contextos particulares a la hora de evaluar si utilizarían una frase u otra en una situación dada. De este modo, son capaces de diferenciar formas de habla en contextos formales como informales. En el caso del grupo A (estrato socio-económico bajo), si bien bajan los índices de uso en el habla con su profesor frente al uso de habla con sus amigos, es clara la mayor utilización que éstos harían en esa situación en particular en contraste con el uso diferenciado que registra el grupo de estrato medio. Así, enunciados del tipo: Se te zafó un tornillo, Se echó el pollo (se fue), Te tienen pa la chocota (te están molestando) presentan un alto índice de uso en ambos grupos en una situación informal. En una situación formal en cambio, el grupo de estrato medio es capaz de diferenciar la inadecuación de uso contextual, a diferencia del grupo de estrato bajo que lo hace en menor medida.

Desde este punto de vista, podemos observar que las diferencias en la evaluación fraseológica presentada a los sujetos de ambos estratos no es sobre si tienen o no conocimiento sobre la forma de habla del otro, sino sobre el manejo situacional que cada grupo hace sobre su forma de habla y sobre el momento de uso de aquellas frases. Un punto que llama bastante la atención es el menor índice porcentual que, en promedio, presentan las mujeres de ambos grupos a la hora de evaluar tanto la producción fraseológica propia, como la perteneciente al grupo social al cual no pertenecen. 


\section{Conclusiones}

En relación con el conocimiento de la forma de habla que cada grupo social tiene sobre el otro y sobre si mismo, podemos sostener que no se presentan mayores dificultades de reconocimiento fraseológico en los individuos. Sin embargo, sí se presentarían diferencias en el uso contextual que cada grupo realizaría en una situación determinada. Según el análisis de los resultados presentados, podemos sostener que el grupo de sujetos de estrato socioeconómico medio tendría lo que H. López Morales (citado por Moreno, 1998) llamaría una mayor conciencia de uso contextual del lenguaje y de adecuación que los jóvenes de estrato socioeconómico bajo. Lo anterior, vendría a dar cuenta de que la existencia de diferentes actitudes lingüísticas supone la aceptación de que los individuos son capaces de evaluar su propio modo de hablar y el discurso de los demás a partir de la presencia de una serie de índices lingüísticos. En este sentido, el grupo de estrato medio expresaría una capacidad para discriminar de forma más clara entre modo de habla formal e informal.

Estos índices, que aparecen codificados socialmente bajo diferentes atributos simbólicos, permiten a los miembros de cualquier comunidad extraer ciertas conclusiones sobre la condición social de los demás, sobre aspectos de su personalidad y sobre la misma situación comunicativa, todo lo cual conduce a planificar de un modo determinado la interacción (Almeida, 2003:187). En este sentido, la importancia de las actitudes viene dada, entre otras cosas, porque pueden condicionar la selección de unas variantes lingüísticas en detrimento de otras, particularmente cuando se tiene en cuenta el contexto de situación (Labov, 1972/1983: 184).

En relación con lo anterior, no deja de llamar la atención que el grupo femenino discrimine en mayor proporción que los hombres de ambos estratos societales. En este sentido, las mujeres de estrato medio, al diferenciar en mayor medida el uso de habla en contextos particulares, considerarían tanto sus frases hechas como las pertenecientes al otro grupo ligadas al ámbito de lo informal. En el caso de sus pares de estrato bajo, si bien aquellas discriminan menos que los hombres de estrato medio, lo hacen de todos modos en mayor proporción que los hombres de su mismo grupo social. Así, podemos decir que los hombres usan las variantes no estándares más que las mujeres, independientemente del estrato social al que se pertenezca (Almeida, 2003).

De igual modo, podemos sostener que las mujeres usan las variantes lingüísticas de mayor prestigio con más frecuencia que los hombres. 
Divergencias y encuentros locutivos en el habla de dos grupos sociales / César Marcelo Díaz Pacheco

En el caso de la muestra, esta conducta es mucho más marcada en el sub-grupo de mujeres de estrato medio. La mayor sensibilidad de las mujeres hacia normas de conducta "correcta" puede ser explicable por el hecho de que tienden más hacia lo conservador, a diferencia del habla masculina que tiende hacia a una forma de habla quizás más tosca (Silva, 2001). Desde este punto de vista, la diferenciación lingüística según el sexo refleja una tendencia general a considerar aceptable o apropiado que los hombres rompan las reglas y que se comporten de manera más ruda, agresiva y vulgar (Silva, 1989:70). Así, el típico doble estándar se aplica a las mujeres, cuyo comportamiento se espera que sea más cortés, más indeciso, más correcto y ajustado a las normas impuestas por la sociedad.

Por otra parte, en cuanto a la evaluación que los sujetos hacen respecto de cuán groseras encuentran las frases hechas de sus pares del otro estrato -y también las propias- podemos decir que los jóvenes del grupo de estrato medio observan con mayor carga disfémica la forma de habla perteneciente al grupo de estrato bajo y la producción fraseológica coincidente entre ambos (frases neutras). En este sentido, el sujeto de clase media al verse poseedor de un código lingüístico más amplio que el sujeto de clase baja, puede transmitir sus experiencias peculiares y realizar juicios de valoración con mayores niveles de conciencia. Su concepto de su propio "yo", a diferencia del sujeto de estrato bajo -poseedor de un código lingüístico más restringido- está verbalmente diferenciado hasta el punto de poder ser él objeto de actividad perceptual (Bernstein, 1964: 370). En el caso del joven de estrato socio-económico bajo -que sólo tiene acceso al código de tipo restringido-, este tenderá a desarrollarse sólo de acuerdo a las reglas propias de su código: su habla no llega a convertirse en el objeto de una actividad perceptiva especial.

Así, pareciera desprenderse de los rasgos expuestos anteriormente que por medio de los usos lingüísticos los individuos son capaces de expresar diversas actitudes y valores culturales relacionados con la posición social que ocupan en la comunidad. En este sentido, estas actitudes y valores pueden ser asumidos por aquellos sujetos en función del tipo de identidad social que quiera ser proyectada (por ejemplo, presentarse o no como una persona de estatus, afirmar o negar los valores comunitarios, identificarse con un lugar a través del habla, etc.). Pero no es menos cierto también que, sobre todo en contextos y situaciones muy precisas, existen ciertas presiones para el empleo de determinadas formas de lenguaje (Almeida, 2003). Estas presiones pueden ser ejercidas tanto desde el grupo al que se pertenece con el fin de mantener la cohesión 
grupal (como cuando se requiere la manifestación de cierto grado de lealtad lingüística a través del empleo de las formas vernáculas de habla), como desde el exterior del grupo con el fin de mantener unas normas de interacción que permitan cierto grado de fluidez intergrupal (por ejemplo, cuando se requiere en determinados contextos situacionales que se abandonen las formas vernáculas de habla y se empleen variedades más estándares).

Por otro lado, cabe preguntarse si las unidades de la fraseología pueden considerarse legítimamente variables sociolingüísticas (Moreno, 1998) con variantes representativas, como lo son las variables fonético-fonológicas o las morfo-sintácticas. Creemos que este estudio ha demostrado que este nivel es válido para comprender el fenómeno de la variación correlacionado con la clase social, a pesar de que se harían necesarios estudios que involucraran grupos más diversos e incluso grupos de diferentes variables geográficas. En este sentido, una de las limitantes de esta investigación considera que la muestra utilizada está constituida por un grupo social reducido, por lo tanto, se establecen conclusiones preliminares y no universales de la variación sociolingüística de las frases hechas. De este modo, sería interesante poder ampliar la muestra en términos geográficos y de cantidad de sujetos participantes con el fin de tomar todos los estratos presentes en la región (bajo, medio y alto) para así, hacer mucho más representativa la muestra.

Finalmente, el estudio presentado puede tener un alcance en el área de la educación. Por un lado, sabemos lo importante que resulta para los estudiantes mantener comportamientos grupales homogéneos, especialmente durante la adolescencia, un período crítico en lo que respecta a la conformación de la identidad social de los individuos (Almeida, 2003:204). Por otro lado, en tanto que una de sus funciones principales es la formación de las personas, el carácter regulativo de la institución escolar lleva a la organización de los centros a la adopción de normas de comportamiento homogéneas que, naturalmente, terminan por chocar con los intereses grupales de los escolares. En este sentido, el acervo lingüístico que el adolescente lleva a la escuela puede ser actualizado a través de la incorporación de rasgos de habla similares y distintos a los de la familia a la que se pertenece, un proceso que se podría llevar a cabo sobre todo durante la pre adolescencia y la adolescencia producto de los cambios que se desarrollan en la personalidad de los individuos y gracias a la influencia que en un momento determinado tiene su grupo de iguales (Labov, 1964). De este modo, esta incorporación de rasgos lingüísticos debiera ser potencial para el desarrollo de la competencia 
Divergencias y encuentros locutivos en el habla de dos grupos sociales / César Marcelo Díaz Pacheco

meta-pragmática de los estudiantes con el fin de poder construir mayores niveles de conciencia que puedan estimular una mejor funcionalidad y adecuación contextual del uso del lenguaje en los individuos.

\section{Bibliografía}

Almeida, M. (2003). Sociolingüística: Introducción a la lingüística actual. Segunda Edición. Universidad de La Laguna, Tenerife.

Allan K., Burridge K. (1991). Euphemism and dysphemism; language used as shield and weapon. Oxford. Oxford University.

Belinchón, M. (1999). Lenguaje no literal y aspectos pragmáticos de la comprensión. En M. de Vega y F. Cuetos (Eds.), Psicolingüística del español (pp. 307-73). Madrid: Editorial Trotta.

Bernstein, B. (1964). Códigos amplios y restringidos: Sus orígenes sociales y algunas consecuencias. Publicación especial del American Anthropologist 66, núm. 6, parte 2.

Corpas Pastor, G. (1996/1997). Manual de fraseología española. Madrid: Gredos.

Coseriu, E. (1973). Sincronía, diacronía e historia; El problema del cambio lingüístico. Madrid: Gredos.

Crespo, N. y Cáceres, P. (2006). La comprensión oral de las frases hechas: Un fenómeno de desarrollo tardío del lenguaje. RLA. Revista de Lingüística Teórica y Aplicada. Disponible en: http://www.scielo.cl/pdf/rla/v44n2/art06.pdf

; Alfaro, P. y Pérez, D. (2008). ¿Cómo comprenden los niños las locuciones? Posibles influencias de la Transferencia y la Familiaridad. Disponible en: http://www.onomazein. net/17/locuciones.pdf

Chamizo, M. (2005). La metáfora (Semántica y pragmática). Disponible en: http://ensayistas.org/critica/retorica/chamizo

Labov, W. (1964). Stages in the acquisition of Standard English. En Shuy, ed., 1964. Pg. 77-103.

(1972/1983). Modelos lingüísticos. Madrid: Cátedra.

(1990). The intersection of sex and social class in the course of linguistic chance. Language Variation and Chance, 2. Pg. 2005-254.

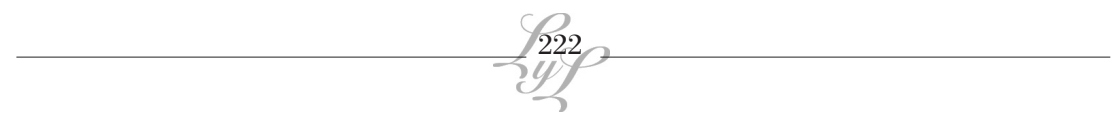


Liang, S. (1991-1992). À propos du dictionnaire français-chinois des collocations françaises. En Cahiers de lexicologie 59, 151-167.

López, H. (1983). Introducción a la lingüística general. Madrid: Playor (Pg. 150).

Matus, R. (1999). Nuestra lengua; ¿Su madre o su Señora madre? El nuevo Diario. Managua, Nicaragua. Disponible en: http:// archivo.elnuevodiario.com.

Moreno Fernández, F. (1998). Principios de Sociolingüística y Sociología del lenguaje. Barcelona: Ariel. 1998.

Navarro, C. (2004). Didáctica de las unidades fraseológicas. Sección Pragmática cultural. Universidad de Verona. Disponible en: http:// www.edinumen.es

Nippold, M. (1998). Later language Development The school-age and adolescent years. Texas: Pro-ed.

Silva Corvalán, C. (1989). Sociolingüística; Teoría y análisis. Madrid: Editorial Alhambra.

Silva Corvalán, C. (2001). Sociolingüística y pragmática del español. Georgetown University Press.

St. Clair, R. (1982). From social history to language attitudes. En Ryan y Giles, eds., 1982. Pg. 164-174. 